\title{
Adenosine abolishes MTX-induced suppression of osteoclastogenesis and inflammatory bone destruction in adjuvant-induced arthritis
}

\author{
Junpei Teramachi ${ }^{1,2}$, Akiko Kukita ${ }^{3}$, Yin-Ji Li ${ }^{1}$, Yuki Ushijima ${ }^{4}$, Hiroshi Ohkuma ${ }^{4}$, Naohisa Wada ${ }^{5}$, \\ Toshiyuki Watanabe ${ }^{1}$, Seiji Nakamura ${ }^{2}$ and Toshio Kukita ${ }^{1}$
}

\begin{abstract}
Methotrexate (MTX) is widely utilized for the treatment of patients with rheumatoid arthritis (RA); however, recent observation of the MTX-resistant patients proposed some difficulty in MTX-dependent therapeutic approach for RA. To access cellular events related to MTX resistance in RA in respect to inflammatory bone destruction, we investigated on an involvement of the potent inflammatory mediator adenosine in the regulation of osteoclastogenesis and inflammatory bone destruction. In rats with adjuvant-induced arthritis (AA rats), MTX efficiently suppressed bone destruction when it was administrated within 3 days after adjuvant injection, while it could not suppress inflammatory bone destruction if MTX was injected at the time of onset of inflammation (at day 10 after adjuvant injection). Time-course change in the level of plasma adenosine of AA rats was estimated by use of high-performance liquid chromatography and elucidated that adenosine level was markedly elevated till 10 days after adjuvant injection. In vitro bone marrow culture system for evaluating osteoclastogenesis, MTX markedly suppressed osteoclastogenesis in a stromal cell-dependent manner. This MTX-induced suppression of osteoclastogenesis was abrogated by the addition of adenosine. MTX suppressed the expression of mRNA for the receptor activator NF- $\kappa$ B ligand (RANKL), but it did not suppress the expression of osteoprotegerin (OPG). The addition of MTX and adenosine together markedly suppressed the level of OPG expression.

Abolishment of MTX action by adenosine was significantly blocked by MRS1754, a highly selective antagonist for the $A_{2 b}$ adenosine receptor $\left(A_{2 b} A R\right)$, but not by caffeine, an antagonist for $A_{1}, A_{2 a}, A_{3} A R\left(A_{1} A R, A_{2 a} A R\right.$, and $\left.A_{3} A R\right)$, which suggests that adenosine acts through $A_{2 b} A R$. Immunohistochemical studies showed abundant expression of $A_{2 b} A R$ in cells localized in the bone-bone marrow boundary of the distal tibia in AA rats but not in control rats. When adenosine was injected in the ankle joints of MTX-treated AA rats, the suppressive effects of MTX on bone destruction was abolished. The current data therefore suggest that upregulation of adenosine production abolished the suppressive effect of MTX on osteoclastic bone destruction. Involvement of the adenosine- $\mathrm{A}_{2 \mathrm{~b}} \mathrm{AR}$ system may explain MTX resistance in RA.

Laboratory Investigation (2011) 91, 719-731; doi:10.1038/labinvest.2011.9; published online 21 February 2011
\end{abstract}

KEYWORDS: adenosine; adenosine receptor A2b; adjuvant arthritis; bone destruction; HPLC; methotrexate; osteoclast; osteoprotegerin

Rheumatoid arthritis (RA) is a chronic inflammatory disease characterized by the destruction of articular cartilage and bone. Bone destruction is mediated by the multinucleated giant cells, osteoclasts. ${ }^{1-3}$ Abundant expression of inflammatory cytokines upregulates osteoclastogenesis within the inflamed regions. Methotrexate (MTX), a monomethylated aminopterin, is widely used and is one of the most effective therapeutics for the treatment of RA. MTX and aminopterin are folic acid antagonists that act as competitive inhibitors of dihydrofolate reductase, a key enzyme of 'de novo' nucleic acid synthesis. These agents were originally developed for use in chemotherapeutic treatment of infant leukemia to inhibit

\footnotetext{
${ }^{1}$ Department of Molecular Cell Biology and Oral Anatomy, Kyushu University, Fukuoka, Japan; ${ }^{2}$ Division of Maxillofacial Diagnostic and Surgical Science, Department of Oral and Maxillofacial Oncology, Faculty of Dental Science, Kyushu University, Fukuoka, Japan; ${ }^{3}$ Department of Microbiology, Faculty of Medicine, Saga University, Saga, Japan; ${ }^{4}$ Department of Research and Development, Kumamoto Flour Milling Company, Kyushu University, Fukuoka, Japan; ${ }^{5}$ Department of Endodontology and Operative Dentistry, Faculty of Dental Science, Kyushu University, Fukuoka, Japan

Correspondence: Professor T Kukita, PhD, Department of Molecular Cell Biology and Oral Anatomy, Faculty of Dental Science, Kyushu University, 3-1-1 Maidashi, Fukuoka 812-8582, Japan.

E-mail: kukitat@dent.kyushu-u.ac.jp

Received 28 June 2010; revised 10 December 2010; accepted 10 December 2010
} 
proliferation of tumor cells. ${ }^{4-6}$ MTX continues to be widely used for chemotherapy of cancer. Low-dose therapy with MTX has been shown to be effective for the treatment of $\mathrm{RA}^{7-9}$ and has become the gold standard for RA. However, some lines of evidence have demonstrated that MTX fails to suppress bone destruction in some RA patients. ${ }^{9,10}$ The presence of MTX resistance reduces the efficacy of MTXdependent therapy. However, the molecular and cellular mechanism of MTX resistance in inflammatory bone destruction remains ambiguous. ${ }^{11}$

Receptor activator NF- $\kappa \mathrm{B}$ ligand (RANKL) is the major cytokine for osteoclastogenesis. RANKL binds to its specific receptor RANK expressed on osteoclast precursors and induces differentiation into multinucleated osteoclasts. ${ }^{12}$ RANKL-induced osteoclastogenesis is blocked by a decoy receptor of RANKL, osteoprotegerin (OPG) ${ }^{13-15}$ Therefore, the local RANKL/OPG ratio determines the potency of osteoclastogenesis in bone tissues.

Adenosine is a key endogenous molecule, which regulates tissue function by activating G-protein-coupled adenosine receptors (ARs). ${ }^{16}$ So far, four types of $A R s, A_{1} A R, A_{2 a} A R$, $\mathrm{A}_{2 \mathrm{~b}} \mathrm{AR}$, and $\mathrm{A}_{3} \mathrm{AR}$ have been identified. $\mathrm{A}_{1} \mathrm{AR}, \mathrm{A}_{2 \mathrm{a}} \mathrm{AR}$, and $A_{3} A R$ are high-affinity receptors, which can be activated by physiological concentrations of extracellular adenosine of $<1 \mu \mathrm{M}$. However, in contrast, $\mathrm{A}_{2 \mathrm{~b}} \mathrm{AR}$ is a low-affinity receptor, which requires adenosine levels that exceed $10 \mu \mathrm{M}$ for activation. In inflammatory disease, it is known that the local adenosine level may rise to pathophysiological concentrations, enabling activation of the low-affinity receptor, $\mathrm{A}_{2 \mathrm{~b}} \mathrm{AR}$.

Aminopterin, an unmethylated form of MTX, is routinely used for selection of B-cell hybridomas in the production of monoclonal antibodies. During the course of our trial to develop novel antibodies affecting osteoclastogenesis, we have accidentally noticed that osteoclastogenesis is markedly inhibited by aminopterin and its monomethylated derivative, MTX (unpublished observations). This paper focuses on investigating the basic cellular events underlying MTX resistance in osteoclastic bone destruction. In vitro experiments demonstrated that suppression of osteoclastogenesis by MTX was abolished by treatment with adenosine, possibly in an $\mathrm{A}_{2 \mathrm{~b}} \mathrm{AR}$-dependent manner, through suppressing $\mathrm{OPG}$ expression. In vivo studies using AA rats also suggested a role of adenosine as an important modulator of bone destruction.

\section{MATERIALS AND METHODS Materials}

Heat-killed Mycobacterium butyricum and mineral oil were obtained from Difco Laboratories (Detroit, MI, USA). RANKL was purchased from Peprotech EC (London, UK). $1 \alpha, 25$-Dihydroxy vitamin $\mathrm{D}_{3}\left(1 \alpha, 25(\mathrm{OH})_{2} \mathrm{D}_{3}\right)$ was from Biomol Research Laboratories (Plymouth Meeting, UK). MTX, adenosine, cytidine, guanosine, uridine, deoxyadenosine, deoxycytidine, deoxyguanosine, thymidine, caffeine, MRS1754, and Leukocyte acid phosphatase kits were obtained from Sigma (St Louis, MO, USA). $\alpha$-Minimum essential medium ( $\alpha$ MEM; cat\#12000-022) and $\alpha$ MEM containing nucleosides ( $\alpha$ MEM + Nuc; cat\#11900-024) and penicillin-streptomycin were purchased from Invitrogen (Grand Island, NY, USA).

\section{Animals and Animal Experiments and Ethics Committee Approval}

Sprague Dawley (SD) rats, Lewis rats were obtained from Kyudo (Tosu, Japan). All animal experiments were performed according to the guidelines for 'Care and Use of Animals of Kyushu University'.

\section{Measurement of Plasma Adenosine Concentration by HPLC}

Peripheral blood was obtained from aorta of AA rats and immediately centrifuged 800 r.p.m. at $4{ }^{\circ} \mathrm{C}$ for $5 \mathrm{~min}$. Supernatants (plasma sample) were immediately frozen and kept in liquid nitrogen and maintained in it till HPLC analysis. After thawing the frozen sample, $90 \mu \mathrm{l}$ samples were mixed with $10 \mu \mathrm{l}$ of $60 \%$ perchloric acid followed by being mixed immediately by vortex. After centrifuge ( 15000 r.p.m., 5 min, $4{ }^{\circ} \mathrm{C}$ ), $50 \mu$ l supernatant was subjected to HPLC (Mighty sil RP-18GP column $4.6 \mathrm{~mm} \mathrm{ID} \times 250 \mathrm{~mm}$ ) (Kantoh Chemical, Tokyo, Japan). Elution was performed by $10 \mathrm{mM}$ potassium phosphate $(\mathrm{pH} 2.9)$ and $1-8 \%$ of acetonitrile at the flowrate of $1.0 \mathrm{ml}$ per second. Elution pattern was monitored by detecting UV absorbance at $260 \mathrm{~nm}$. Various quantities of standard adenosine were also injected to HPLC to make a standard curve for the quantification of the sample.

\section{Bone Marrow Culture System}

Formation of osteoclast-like multinucleated cells (MNCs) from rat bone marrow cells was performed as described previously. ${ }^{17,18}$ Briefly, bone marrow cells were obtained from the tibia and femur of 4-6-week-old male SD rats. Cells were seeded into 24 -well culture plates $\left(10^{6}\right.$ cells/well $)$ and cultured for 4 or 5 days in $\alpha$ MEM without or with nucleosides $(\alpha \mathrm{MEM}+\mathrm{Nuc})$ containing $15 \%$ FBS in the presence of $10^{-8} \mathrm{M} 1 \alpha, 25(\mathrm{OH})_{2} \mathrm{D}_{3}, 10 \%(\mathrm{v} / \mathrm{v})$ heat-treated ROS17/2.8 cell-conditioned medium (ht-ROS CM) and various concentrations of MTX. Cells were fixed with acetone-citrateformaldehyde, and stained for TRAP using a leukocyte acid phosphatase kit. TRAP-positive cells containing more than three nuclei were counted as osteoclast-like MNCs. For stromal cell-free cultures, bone marrow stromal cells were depleted by Sephadex ${ }^{\mathrm{TM}}$ G-10 (GE Healthcare, Uppsala, Sweden) column as described previously. ${ }^{18}$ The non-adherent bone marrow cells were cultured in the presence of $10^{-8} \mathrm{M}$ $1 \alpha, 25(\mathrm{OH})_{2} \mathrm{D}_{3}, 20 \mathrm{ng} / \mathrm{ml}$ sRANKL and $10 \%$ (v/v) htROSCM. Cells were cultured in 96-well culture plates $\left(4 \times 10^{5}\right.$ cells/ well) in the presence of various concentrations of MTX. After 4 days of culture, the cells were fixed and stained with TRAP. 


\section{Preparation of Rat Primary Osteoblasts}

Rat primary osteoblasts were prepared by sequential digestion of calvarias of 2-day-old SD rats with collagenase and dispase, according to the method of Takahashi et al ${ }^{19}$ and Kukita et al. ${ }^{18}$

\section{Semiquantitative RT-PCR}

Total cellular RNA was extracted from cultured cells using TRIzol Reagent (Invitrogen, Carlsbad, CA, USA) and subjected to semiquantitative RT-PCR using RT-PCR kit (Takara, Kyoto, Japan) according to the manufacturer's protocol. The primers used for PCR were as follows: rat RANKL forward, CGT TTG CTC ACC TCA CAA TC; rat RANKL reverse, ACG CTA ATT TCC TCA CCA GC; rat OPG forward, ATT GGC TGA GTG TTC TGG TG; rat OPG reverse, GCC CAG TGA CCA TTC CTA AC; rat cathepsin K forward, TCT CTC GGC GTT TAA TTT GG; rat cathepsin K reverse, CAC ATA TGG GAA AGC ATC TTC A; rat $A_{1} A R$ forward, CAT TGG GCC ACA GAC CTA CT; rat $A_{1} A R$ reverse, CAA GGG AGA GAA TCC AGC AG; rat $\mathrm{A}_{2 \mathrm{a}} \mathrm{AR}$ forward, CTT CGC TAT CAC CAT CAG CA; rat $\mathrm{A}_{2 \mathrm{a}} \mathrm{AR}$ reverse, AAA TTG CAA TGA TGC CCT TC; rat $\mathrm{A}_{2 \mathrm{~b}} \mathrm{AR}$ forward, CTT TCA CAG CTG CCT CTT CC; rat $\mathrm{A}_{2 \mathrm{~b}} \mathrm{AR}$ reverse, AGT CAG TCC AAT GCC AAA GG; rat actin forward, GGG TGT GAT GGT GGG AAT G; rat actin reverse, GCT CGA AGT CTA GAG CAA CAT AGC; rat GAPDH forward, GGT GAT GCT GGT GCT GAG TA; rat GAPDH reverse, ACT GTG GTC ATG AGC CCT TC. The PCR products were subjected to electrophoresis on $1.5 \%$ agarose gels and visualized by ethidium bromide staining with UV light illumination.

\section{Induction of Adjuvant Arthritis in Lewis Rats and X-Ray Imaging}

Adjuvant arthritis was induced as described previously. ${ }^{20,21}$ Briefly, 5-week-old female Lewis rats were intradermally injected at the base of the tail with complete adjuvant consisting of $25 \mathrm{mg} / \mathrm{kg}$ heat-killed M. butyricum (Difco Laboratories) suspended in mineral oil. In control experiments, rats were injected with mineral oil alone. All animals were anesthetized with diethyl ether prior to adjuvant injections. MTX $(1 \mathrm{mg} / \mathrm{kg} /$ week) or vehicle (phosphatebuffered saline (PBS)) was injected intraperitoneally. These injections were performed once a week beginning 3 days after the adjuvant injection. Adenosine or vehicle (PBS) was injected into the ankle-joint cavities of adjuvant-injected rats. Thirty-gauge needles were used to inject $80 \mu \mathrm{g}$ adenosine in $150 \mu \mathrm{l}$ PBS into the left ankle joints. As an internal control, $150 \mu$ l PBS was injected into the right ankle joint. Injections were performed every 3 days beginning on the same day as MTX injections (3 days after the adjuvant injection). The degree of ankle-joint swelling was measured with a digital caliper (Mitutoyo, Nakatsugawa, Japan) at 21 days after the adjuvant injection. The rats were killed on day 21 after the first injection of adjuvant. Hind paws of rats were collected and the level of bone destruction was imaged by using a soft
X-ray analysis system (SOFTEX: SRO-M 50; SOFTEX, Tokyo, Japan) and the ultra-speed radiographic film (Kodak, Rochester, NY, USA). Tissues were then processed for immunohistochemical analysis.

\section{Immunohistochemistry and Histological Analysis}

After fixation by perfusion with $4 \%(\mathrm{w} / \mathrm{v})$ paraformaldehyde/ PBS, tissue blocks were taken from the hind paws (tarsal bones and tibia) of Lewis rats and immersed in the same fixative for $6 \mathrm{~h}$ at $4{ }^{\circ} \mathrm{C}$, followed by washing in PBS overnight at $4{ }^{\circ} \mathrm{C}$. After decalcification in $10 \%(\mathrm{w} / \mathrm{v})$ ethylene diamine tetraacetate for 3 weeks at $4{ }^{\circ} \mathrm{C}$ and dehydration in ethanol series, the tissue blocks were embedded in paraffin. Sections $(6 \mu \mathrm{m})$ of ankle joints including tibia, talus, and calcaneus were prepared and immunostained. After blocking the nonspecific binding sites with $10 \%$ donkey serum and $10 \%$ goat serum for $60 \mathrm{~min}$ at room temperature, sections were incubated with rabbit polyclonal anti-rat $\mathrm{A}_{2 \mathrm{~b}} \mathrm{AR}$ antibody $(20 \mu \mathrm{g} / \mathrm{ml}$; Chemicon, CA, USA). After washing in PBS, sections were incubated with HRP-labeled donkey anti-rabbit IgG (1:200 dilution; Amersham Biosciences, Uppsala, Sweden) for $30 \mathrm{~min}$ at room temperature. Color development was performed using a DAB substrate kit (Vector Laboratories, Burlingame, CA, USA).

\section{Statistical Analysis}

All data are presented as mean \pm s.e.m. Statistical analysis was performed using Student's $t$-test. Data shown are representative results from three or more independent experiments.

\section{RESULTS}

\section{Failure in Suppression of Inflammatory Bone Destruction by Late Stage Administration of MTX in AA Rats}

To investigate the therapeutic effects of MTX on osteoclastic bone destruction and hind paw swelling, rats were given injections intraperitoneally with MTX at different timing after adjuvant injection. As shown in Figure 1, no any clinical symptom was observed in sham-operated rats (Control). Adjuvant injection induced significant inflammation and bone destruction (CFA). MTX suppressed hind paw swelling and bone destruction if administrated 3 days after adjuvant injection (CFA + MTX 3d $\sim)$. However, in contrast, MTX did not suppress inflammation as well as bone destruction if it was administrated after the onset of inflammation (10 days after adjuvant injection) (CFA + MTX 10d $\sim)$.

\section{Significant Increase in the Level of Plasma Adenosine in AA Rats}

Change in the level of adenosine of AA rats was examined by use of HPLC. As shown in Figure 2, the level of adenosine was significantly increased in response to the progress of arthritis. At first 3 days of adjuvant injection, we could only detect low level of plasma adenosine $(0.5-1 \mu \mathrm{M})$. However, 
a

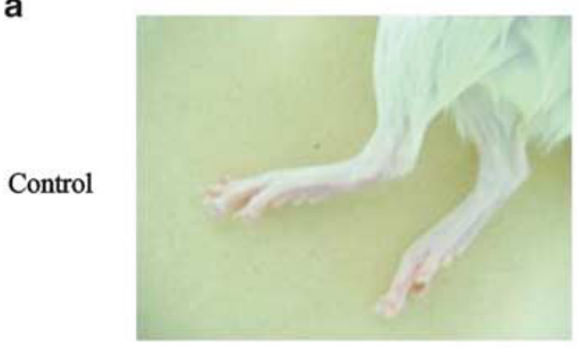

CFA

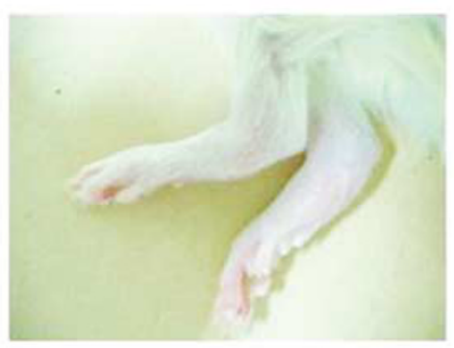

CFA

MTX 3d-

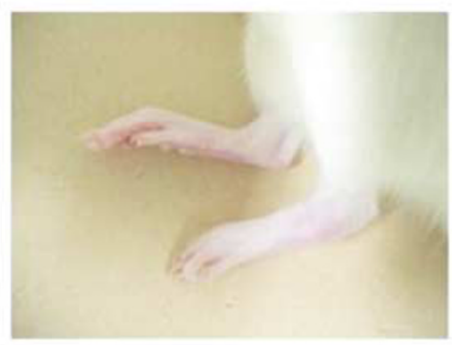

CFA

MTX 10d

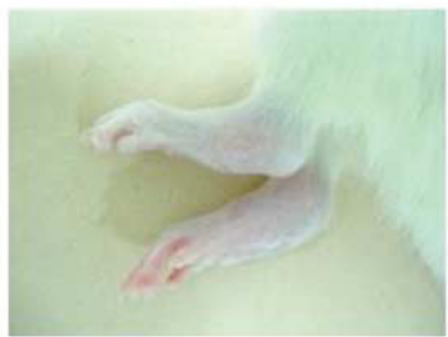

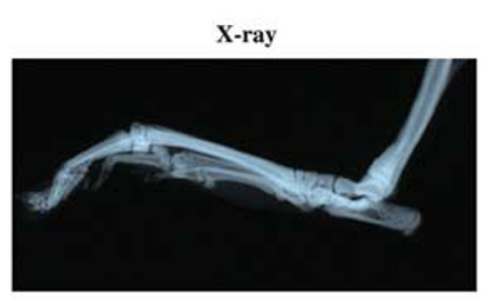
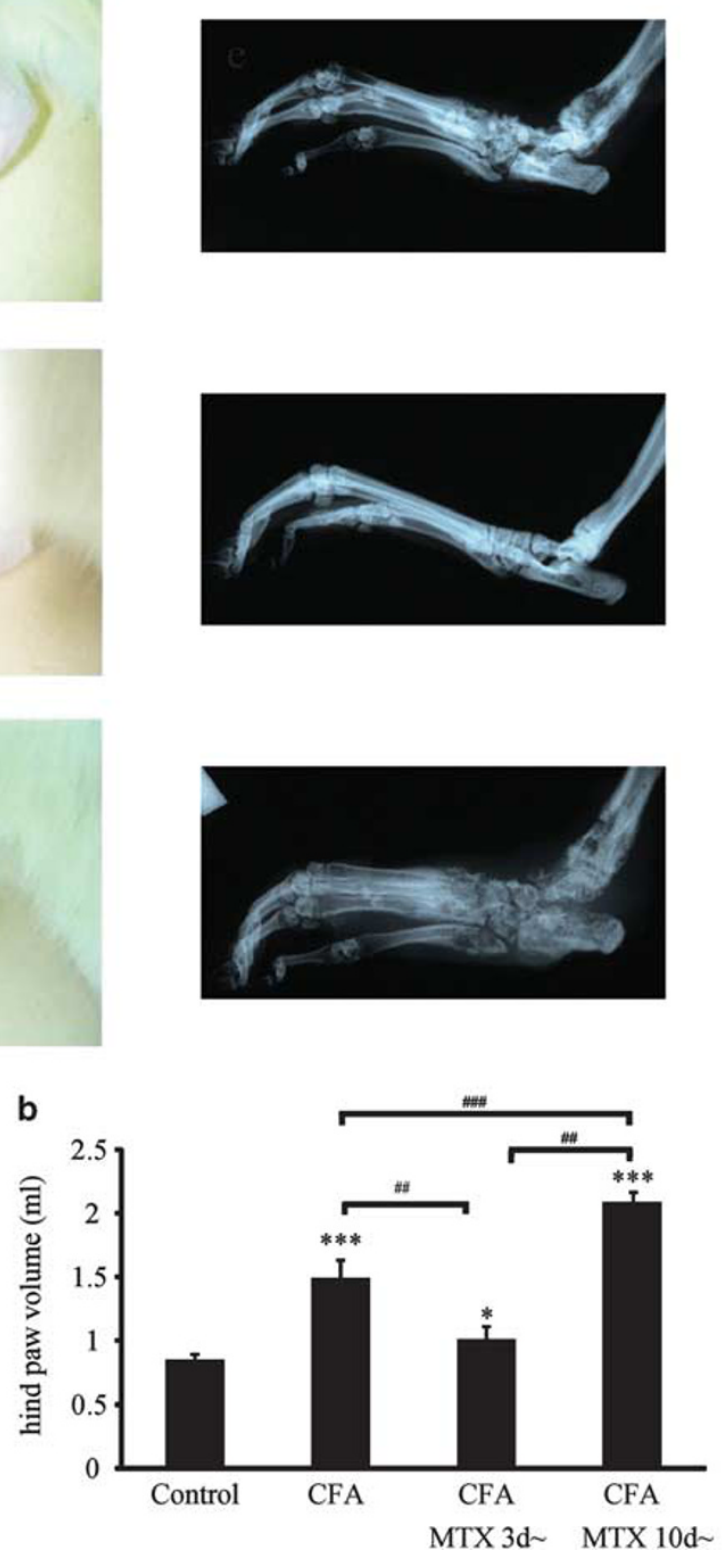

HE
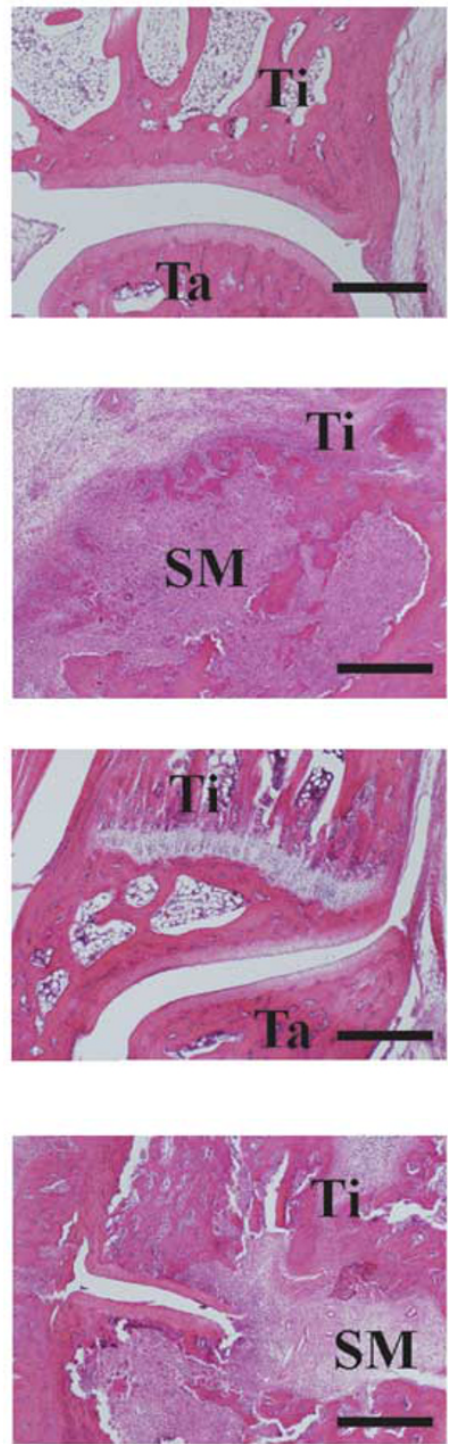

Figure 1 Difference in the therapeutic effects of MTX in AA rat before or after the onset of inflammation. The MTX injections (1 mg/kg/week) were performed intraperitoneally once a week beginning on day 3 (before the onset of inflammation) or day 10 (at the onset of inflammation) after the adjuvant injection. (a) Clinical symptom (left panels), X-ray radiogram (middle panels, X-ray) and histological analysis (right panels, HE) of AA rats treated with MTX. (Control) Sham-operated rats. (CFA) Adjuvant injection induced significant inflammation and bone destruction. (CFA + MTX 3d ) MTX suppressed hind paw swelling and bone destruction if administrated 3 days after adjuvant injection. (CFA + MTX 10d ) MTX was administrated after the onset of inflammation (10 days after adjuvant injection). Ti, tibia; Ta, tarsal bone; SM, synovial membrane. Bars $=200 \mu \mathrm{m}$. (b) Quantitative demonstration of ankle-joint swelling in AA rats. Hind paw swelling was quantitatively measured as described in Materials and methods. Each column and bar represents the mean \pm s.e.m. for eight hind paws of four rats (right and left hind paw for each rats) $(n=8)$. Data were analyzed by Student's $t$-test. ${ }^{\star} P<0.05$ and ${ }^{* * *} P<0.001$ compared with control rats $(n=8)$. ${ }^{\# \#} P<0.01$ and ${ }^{\# \# \#} P<0.001$ when compared each pair. 


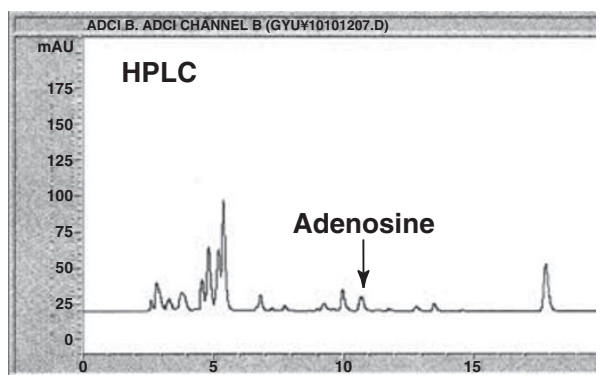

Retention time (min)

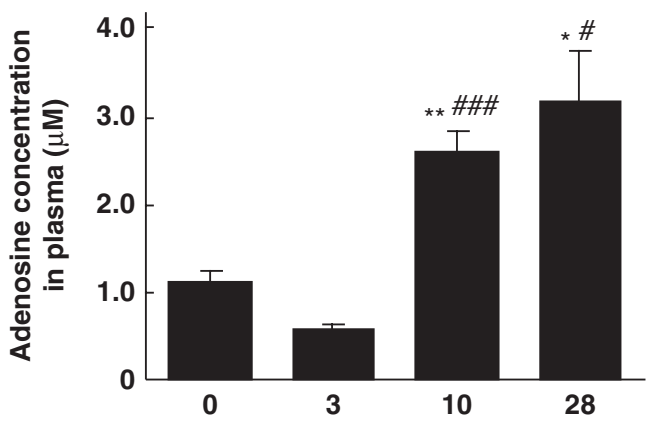

(Days after adjuvant injection)

Figure 2 Marked elevation of plasma adenosine level during the progress of adjuvant arthritis. (Upper panel) Analysis of the plasma sample by HPLC. Plasma samples were obtained and pretreated as described in Materials and methods. Elution profile of the typical sample of rat plasma obtained from AA rats is demonstrated. Position of adenosine peak was demonstrated with an arrow. Vertical line shows absorption in UV $(280 \mathrm{~nm})$. (Lower panel) Time-course change in the plasma adenosine level estimated by use of HPLC. Adenosine concentrations of each plasma samples of various time points were estimated by HPLC. Day 0: before adjuvant injection; Day 3: 3 days after adjuvant injection (with no feature of inflammation); Day 10: 10 days after adjuvant injection (time of the onset of inflammation); Day 28: 28 days after adjuvant injection (the period of severe inflammation and bone destruction). ${ }^{*} P<0.05,{ }^{*} P<0.01$ if compared with data of day 0 . $\# P<0.05$, \#\#\#P<0.001 if compared with data of day 3 .

significant increase in the plasma adenosine was apparent at 10 days. Such high level of plasma adenosine was maintained during the period of severe inflammation and bone destruction.

\section{Adenosine Is Responsive for Abolishment of MTX Action in Osteoclastogenesis}

To address the cellular events concerning such MTXinsensitive observed in vivo, we examined the effect of MTX on osteoclastogenesis in vitro by use of whole bone marrow culture system. As shown in Figure 3a (also in Figure 3d), formation of osteoclast-like MNCs was suppressed by MTX in a dose-dependent manner if the initial culture medium contained no nucleosides and deoxynucleosides. Significant inhibition was apparent at very low concentrations $(1 \mathrm{nM})$ of MTX. As MTX is known to block biosynthesis of nucleosides and deoxynucleosides through inhibiting de novo nucleic acid synthesis, we cultured bone marrow cells in the culture medium containing all nucleosides and deoxynucleosides in order cells could utilize alternative pathway of nucleic acid synthesis, the salvage pathway. Interestingly, osteoclastogenesis was not affected by MTX in the presence of all nucleosides and deoxynucleosides (Figure $3 \mathrm{~b}$ and $\mathrm{d}$ ). These data demonstrated that MTX-mediated inhibition of osteoclastogenesis was completely abolished by the addition of nucleosides and deoxynucleosides in whole bone marrow cultures. We further examined whether MTX-mediated inhibition of osteoclastogenesis occurred through influencing bone marrow stromal cells. As shown in Figure 3c, MTX did not inhibit RANKL-dependent osteoclastogenesis from nonadherent bone marrow cells depleted of stromal cells even in the absence of nucleosides. These data suggest that MTX acts on the bone marrow stromal cells to express inhibitory effects on osteoclastogenesis but it does not directly act on cells in the osteoclast lineage.

As a lack of nucleosides was required for the inhibitory effect of MTX in the bone marrow culture system, we next determined which nucleosides and deoxynucleosides were involved in the abolishment of MTX-induced inhibition of osteoclastogenesis. As shown in Figure 4a, we examined ribonucleosides adenosine, cytidine, guanosine and uridine, and deoxynucleosides deoxyadenosine, deoxycytidine, deoxyguanosine and thymidine. Each nucleoside or deoxynucleoside was added individually to bone marrow cultures of osteoclastogenesis in the presence of MTX at a concentration $(100 \mathrm{nM})$ that completely inhibited osteoclastogenesis, in the absence of nucleosides. The addition of adenosine or deoxyadenosine but not other nucleosides abolished the inhibitory effect of MTX (also in Figure 3d, lower panel). These data strongly suggested that inhibition of osteoclastogenesis by MTX was mediated by blocking adenosine production.

\section{MTX Inhibits RANKL Expression Whereas Adenosine Inhibits OPG Expression}

In order to address the mechanism of MTX-induced inhibition of osteoclast formation in bone marrow cultures, we analyzed expression of RANKL using semiquantitative RT-PCR. As shown in Figure 4b, addition of MTX inhibited expression of RANKL mRNA induced by $1 \alpha, 25(\mathrm{OH})_{2} \mathrm{D}_{3}$. On the other hand, expression of OPG mRNA was not affected by the addition of MTX. Addition of adenosine did not influence the level of RANKL mRNA; however, adenosine strongly inhibited the expression of OPG mRNA (Figure 4b). From these data, it would be possible to consider that MTXinduced suppression of osteoclastogenesis was associated with the suppression of RANKL expression, and that adenosine-mediated recovery of osteoclastogenesis was probably caused by inhibition of OPG expression.

To determine the mechanism by which adenosine inhibits OPG expression, we analyzed expression of the various ARs in rat primary osteoblasts. We analyzed expression of $\mathrm{AR}$ mRNA by RT-PCR as shown in Figure 5a. Among the four 

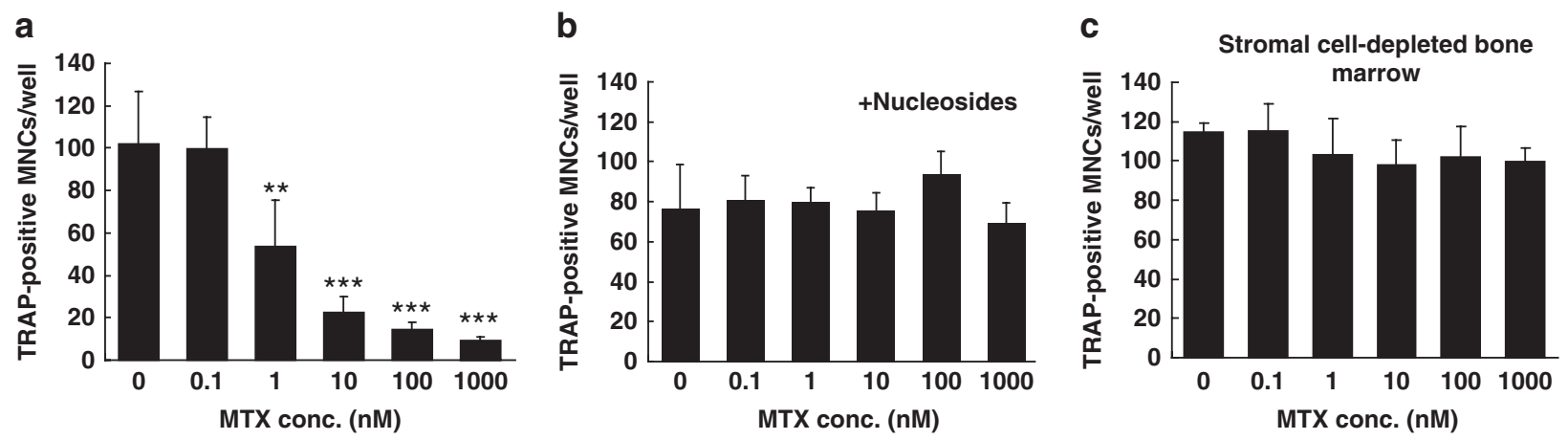

d
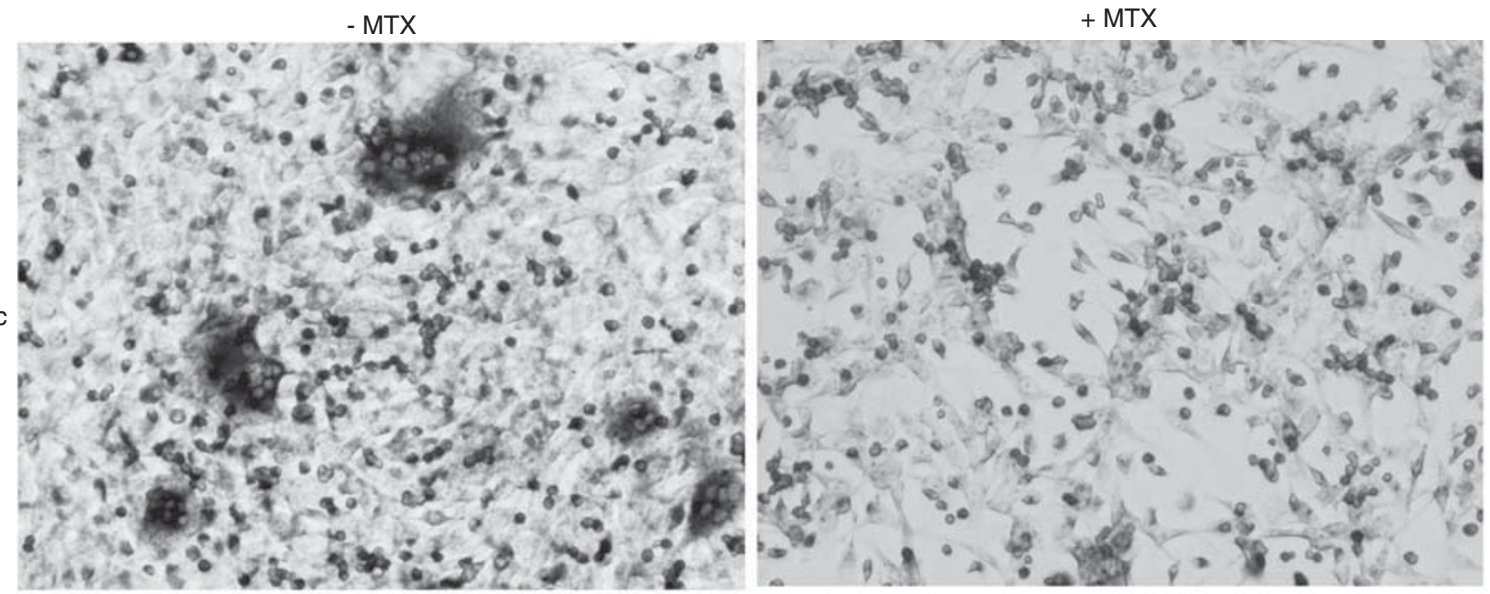

Nuc
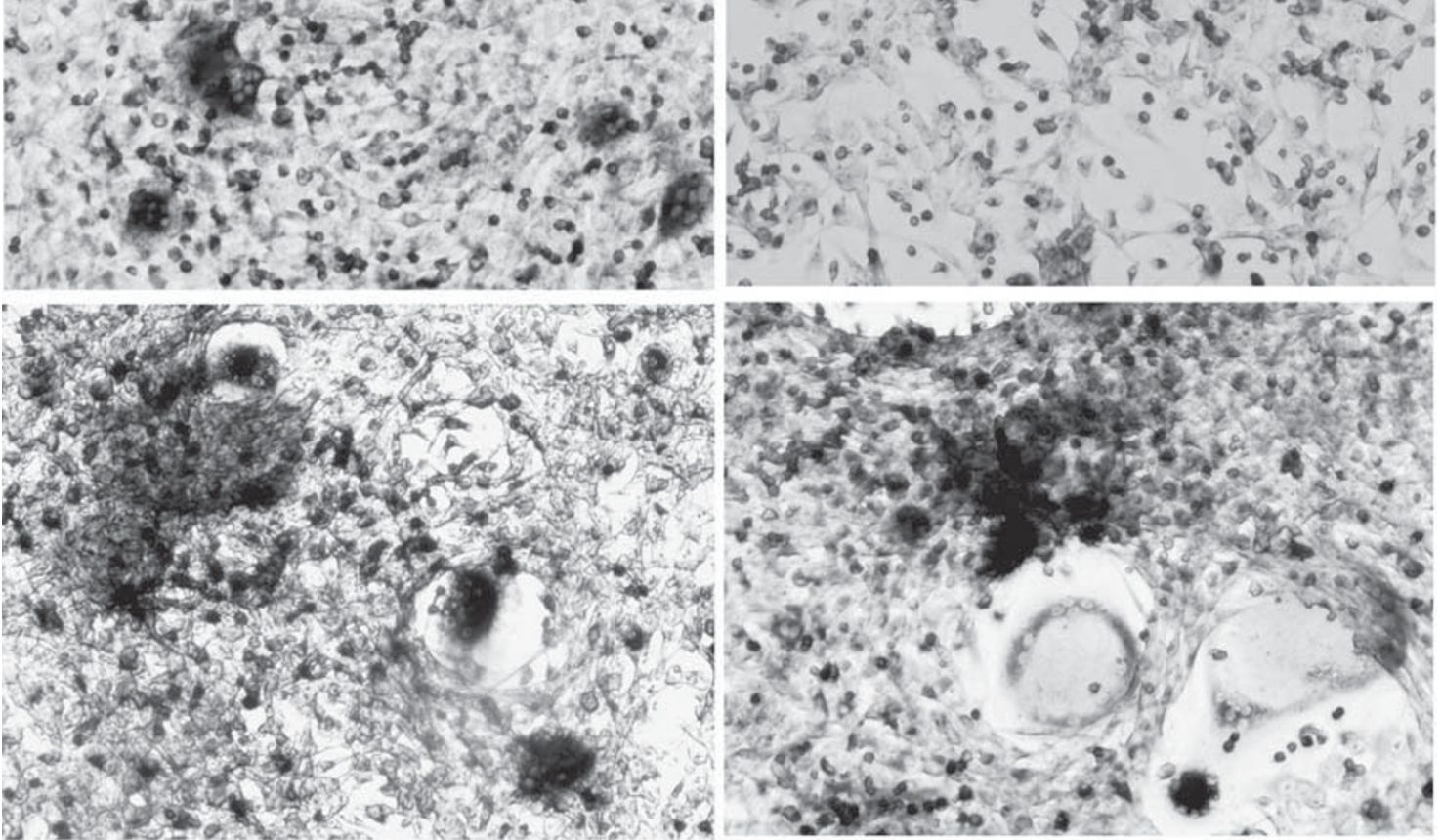

+ MTX + Adenosine

+ MTX + Cytidine
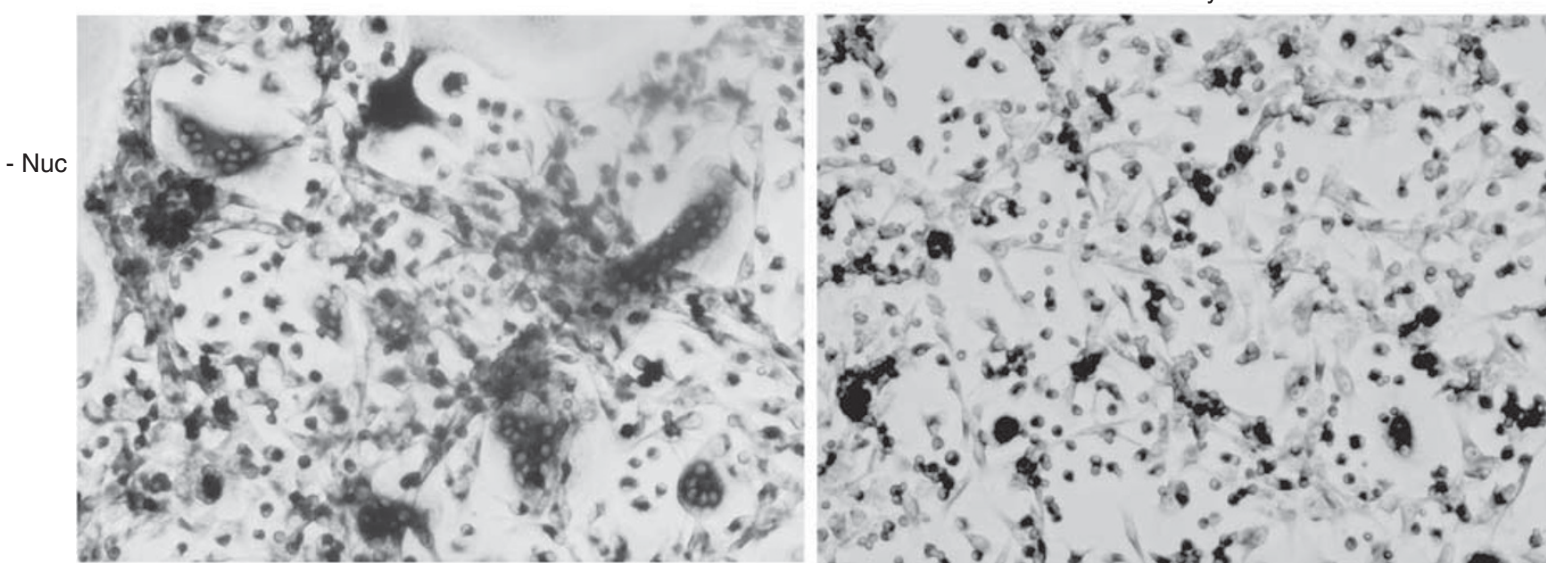
different receptors $\left(A_{1}, A_{2 a}, A_{2 b}\right.$, and $\left.A_{3}\right)$, expression of $A_{1}$, $A_{2 a}$, and $A_{2 b}$ ARs was detected.

\section{Adenosine Inhibits MTX Action through $A_{2 b} A R$}

To determine which receptor was involved in abolishing MTX-induced suppression of osteoclastogenesis, we assessed the effects of AR antagonists. MTX-induced suppression of osteoclastogenesis was not affected by the addition of caffeine, an antagonist of $A_{1} A R, A_{2 a} A R$, and $A_{3} A R$ (Figure $5 b$ ). However, as shown in Figure $5 c$, abolishment of MTXinduced suppression of osteoclastogenesis was blocked by treatment with MRS1754, a highly selective antagonist for $\mathrm{A}_{2 \mathrm{~b}} \mathrm{AR}^{22}{ }^{22}$ MRS1754 itself had no significant effect on osteoclastogenesis (Table 1). These data strongly suggested that the abolishment of MTX-induced suppression in osteoclastogenesis mediated by adenosine was through the low-affinity $\mathrm{AR}, \mathrm{A}_{2 \mathrm{~b}} \mathrm{AR}$. To assess an involvement of $\mathrm{A}_{2 \mathrm{~b}} \mathrm{AR}$ in inflammatory bone destruction, we examined expression of $\mathrm{A}_{2 \mathrm{~b}} \mathrm{AR}$ protein in distal tibia of $\mathrm{AA}$ rats with severe bone destruction around the ankle joints by use of immunohistochemistry. As shown in Figure 5d, a significant number of $\mathrm{A}_{2 \mathrm{~b}} \mathrm{AR}$-positive cells were detected at the bone-

Figure 4 Adenosine or deoxyadenosine abolished MTX-induced suppression of osteoclastogenesis through suppression of OPG mRNA expression. (a) Effect of nucleosides on MTX-induced suppression of osteoclastogenesis. Bone marrow cells were cultured for 4 days in 24-well plates in medium containing $100 \mu \mathrm{M}$ of one of four nucleosides (adenosine, cytidine, guanosine, or uridine) or deoxynucleosides (deoxyadenosine, deoxycytidine, deoxyguanosine, or thymidine) in the presence of $100 \mathrm{nM}$ MTX. Control cells were grown in medium without added nucleosides in the presence or absence of $100 \mathrm{nM}$ MTX. Cells were stained for TRAP, and TRAP-positive MNCs were counted. Data present means \pm s.e.m. from four cultures $(n=4)$. Data were analyzed by Student's $t$-test. ${ }^{* *} P<0.001$ compared with the control culture in the absence of nucleosides. Data are representative of three independent experiments. (b) Effect of MTX and adenosine on the expression of mRNA for RANKL and OPG. Bone marrow cells were cultured for 4 days in 24-well plates in the presence or absence of adenosine, MTX, and $1 \alpha, 25(\mathrm{OH})_{2} \mathrm{D}_{3}$. Cells were analyzed by semiquantitative RT-PCR as described in Materials and methods (Lower panel). Intensity of the bands were quantified and standardized by actin using NIHsion and demonstrated in the lower panel. Similar data were obtained in three independent experiments. Data represent a typical data in three independent experiments. bone marrow interface in the distal tibia of AA rats. No positive cells were observed in the corresponding area of control rats. Normal rabbit IgG (control) showed no staining either in control rats or AA rats. These data show that $\mathrm{A}_{2 \mathrm{~b}} \mathrm{AR}$ is significantly expressed in cells located in the bone-bone marrow interface of the distal tibia of AA rats.

a

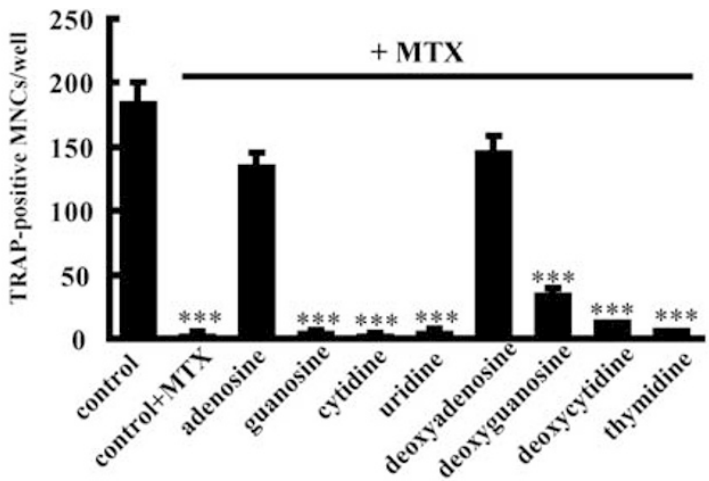

b

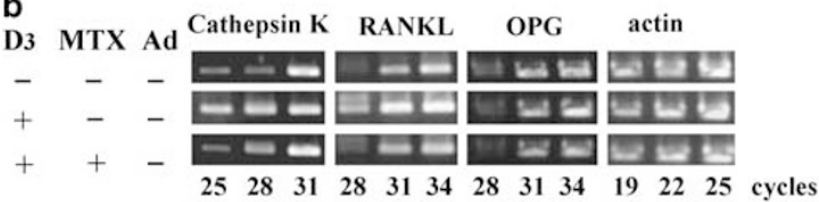

$\begin{array}{cc}\text { D3 } & \text { MTX } \\ + & + \\ + & +\end{array}$
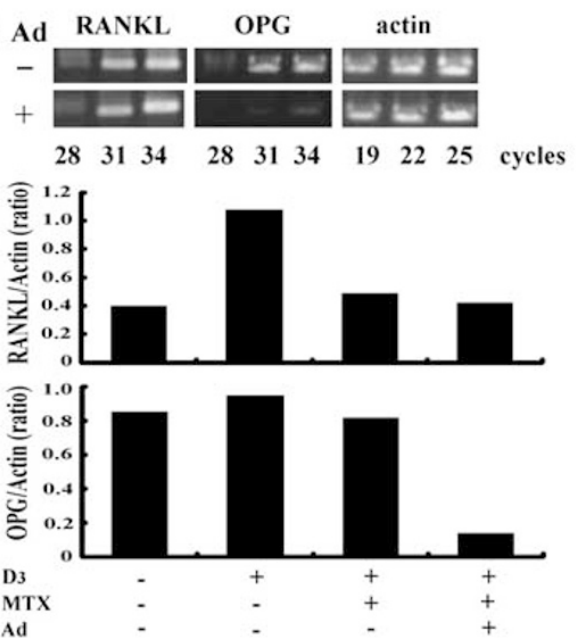

Figure 3 Inhibition of osteoclastogenesis by MTX is caused by lack of nucleosides and mediated by stromal cells. (a) Effect of MTX on osteoclastogenesis in normal medium. Rat bone marrow cells were cultured in the presence of various concentrations of MTX. At 4 days of culture, cells were fixed and stained for TRAP, and TRAP-positive MNCs were counted. (b) Osteoclastogenesis in the presence of exogenous nucleosides and deoxynucleosides. Rat bone marrow cells were cultured in the presence of various concentrations of MTX in culture medium containing nucleosides ( $40 \mu \mathrm{M}$ each nucleoside). At 4 days of culture, cells were fixed and stained for TRAP, and TRAP-positive MNCs were counted. (c) Osteoclastogenesis in stromal cell-free culture. Bone marrow cells were depleted of stromal cells and were cultured as described in Materials and methods in the presence of various concentrations of MTX in culture medium containing no nucleosides. At 4 days of culture, cells were fixed and stained for TRAP, and TRAP-positive MNCs were counted. Data present means \pm s.e.m. from four cultures. Data were analyzed by Student's $t$ test. ${ }^{\star *} P<0.01$ and ${ }^{* * *} P<0.001$ compared with the culture without MTX. Data are representative of three independent experiments. (d) Photographs of TRAP-stained cultures. (Upper panel) Whole bone marrow culture formed in the presence of nucleosides (+ Nuc) or absence of nucleosides (-Nuc) with MTX (+ MTX) or without MTX (-MTX). (Lower panel) In the absence of nucleosides, bone marrow cell cultures were treated with MTX in the presence of adenosine (+ Adenosine) or cytidine (+Cytidine). Original magnification $\times 50$. 


\section{Adenosine Abolishes MTX Influence In Vivo}

In the current paper, MTX-induced suppression of osteoclastogenesis in vitro was abolished by the addition of adenosine to the culture medium. We further examined the possibility that adenosine could abolish MTX-induced suppression of osteoclastogenesis in vivo using AA rats. In
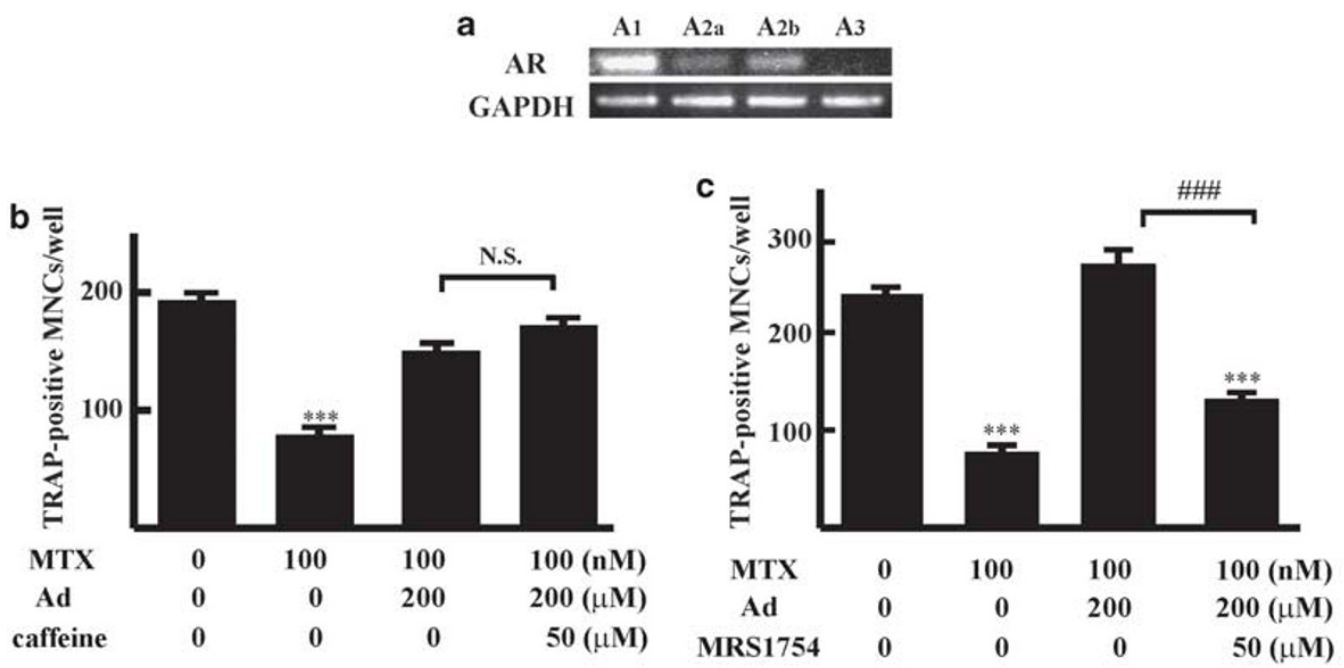

d
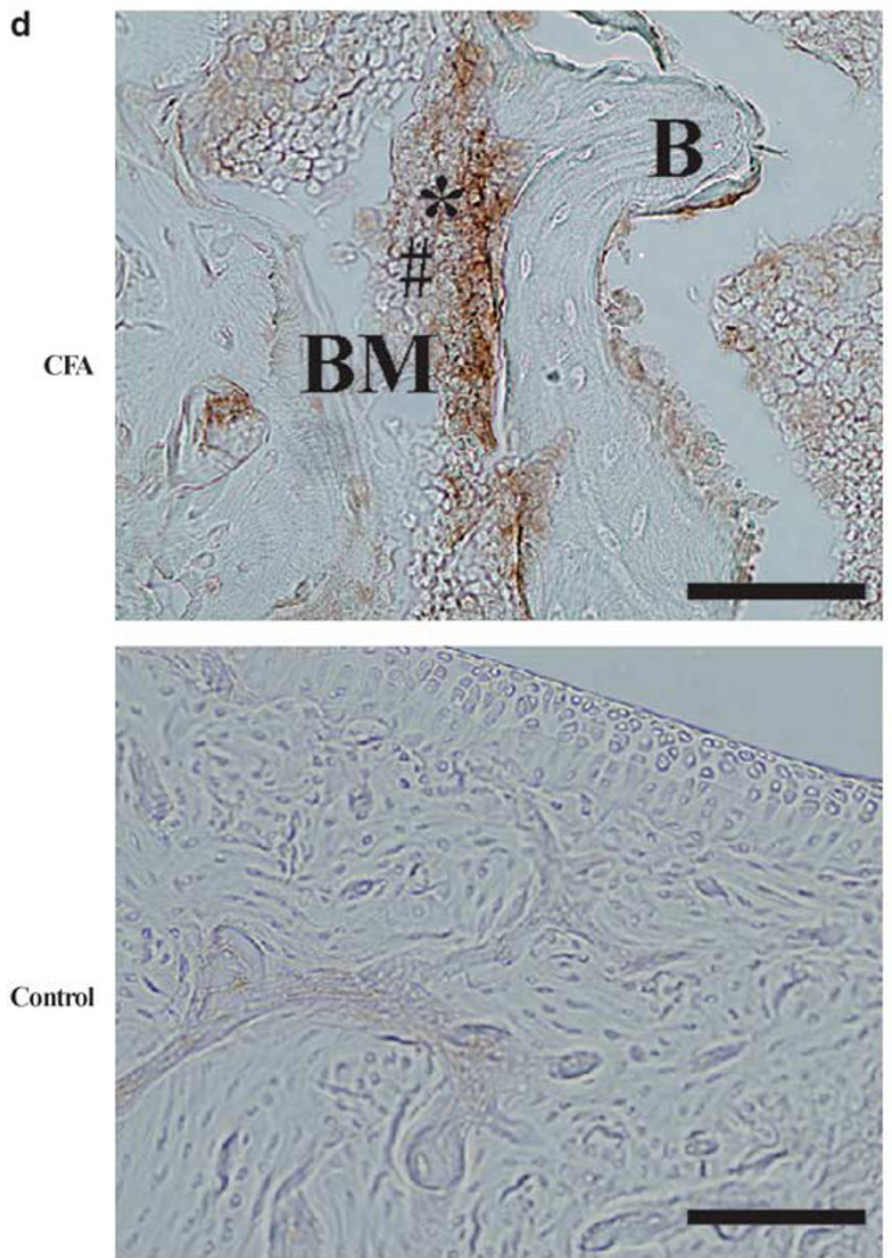

anti-A2bAR
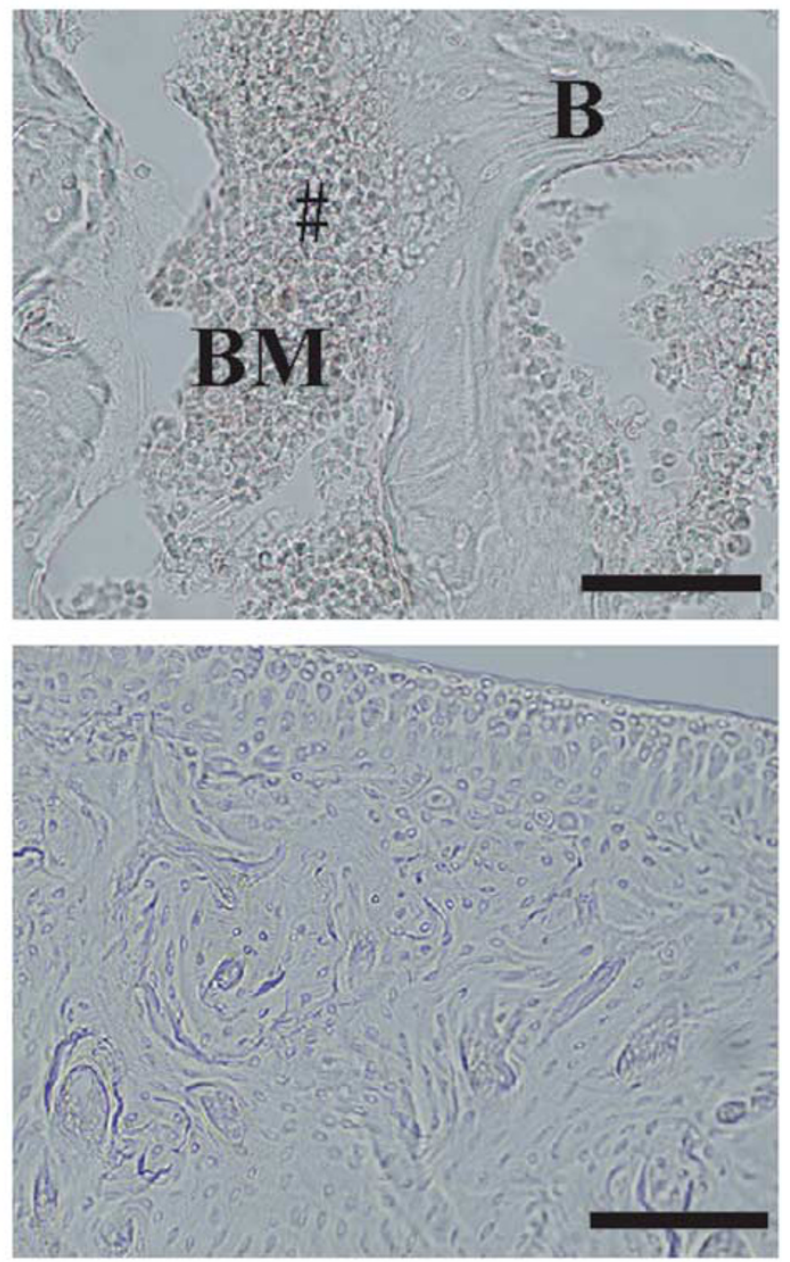

control IgG 
MTX-treated rats, the swelling of the ankle joints induced by adjuvant injection was dramatically decreased (Figure 6a, CFA + MTX, and Figure 6b). When adenosine was injected into the left ankle joints of MTX-treated AA rats, significant hind paw swelling and bone destruction were observed (Figure 6a, CFA + MTX + Ad, and Figure 6b), while MTXinduced suppression of bone destruction was apparent in the right ankle joints only with vehicle injection. These data suggest that high local adenosine concentration at the injection site has abolished the suppressive effect of MTX on inflammation and bone destruction in AA rats. As shown in the X-ray image and histological data, suppression of bone destruction in AA rats induced by the treatment with MTX was abolished by the adenosine treatment. These data suggest an involvement of adenosine in the abolishment of MTXinduced suppression of bone destruction.

\section{DISCUSSION}

In the present study, MTX-induced suppression of bone destruction in vivo was diminished by the addition of adenosine. Our current data suggest that adenosine has a key role in MTX insensitivity. We also discovered that adenosine inhibits OPG mRNA expression and affects osteoclastogenesis through $\mathrm{A}_{2 \mathrm{~b}} \mathrm{AR}$. Adenosine is either released from cells or is formed extracellularly by dephosphorylation of extracellular adenosine triphosphate (ATP), followed by diffusion to cell surface ARs expressed on the surface of adjacent cells. ${ }^{16}$ The level of extracellular adenosine increases at sites of metabolic stress, inflammation, and infection. For example, a recent study has documented that plasma adenosine levels reach $4-10 \mu \mathrm{M}$ in patients with sepsis, a condition associated with both ischemia and inflammation, whereas adenosine concentrations in healthy individuals are $<1 \mu \mathrm{M} .{ }^{16,23}$ In the synovial fluid of patients with RA, adenosine concentration may reach the $10-100 \mu \mathrm{M}$ range. ${ }^{24,25}$ These higher levels of adenosine in inflammation are able to activate the low-affinity receptor, $A_{2 b} A R$. In $A A$ rats with severe bone destruction, the adenosine level is presumed to be increased as a result of the inflammatory process. In the current study, a marked induction of $\mathrm{A}_{2 \mathrm{~b}} \mathrm{AR}$ protein was detected in the area of severe bone destruction in AA rats, suggesting that these areas with severe bone destruction could be regulated by adenosine. Furthermore, caffeine did not abolish suppression of osteoclastogenesis by MTX, suggesting non-involvement of $A_{1}, A_{2 a}$, and $A_{3} A R$ in this process. In contrast, $A_{2 b} A R$ appears to be involved in the regulation of inflammation and osteoclastogenesis in RA. A highly selective $\mathrm{A}_{2 \mathrm{~b}} \mathrm{AR}$ inhibitor, MRS1754, could be a potential seed of medicine for the treatment of inflammation and bone destruction in RA. In our current study, MTX treatment from 3 days after adjuvant injection markedly suppressed bone destruction as well as osteoclast recruitment, presumably because joint tissues express only low levels of adenosine prior to the initiation of inflammation. In other words, MTX could not suppress osteoclastogenesis if synovial tissue contains high levels of adenosine. In concordance with this hypothesis, injection of

\section{Table 1 Involvement of $A_{2 b} A R$ in the recovery of osteoclastogenesis suppressed by MTX}

\begin{tabular}{lccc}
\hline MTX $(\mathrm{nM})$ & Adenosine $(\mu \mathrm{M})$ & MRS1754 $(\boldsymbol{\mu M})$ & TRAP-positive MNC per well mean \pm s.e.m. \\
\hline 0 & 0 & 0 & $192.5 \pm 3.27$ \\
200 & 0 & 0 & $4 \pm 0.70^{* * *}$ \\
200 & 200 & 0 & $140.3 \pm 40.15$ \\
200 & 200 & 50 & $77.7 \pm 18.81^{* * *}$ \\
0 & 0 & 50 & $170.8 \pm 5.18$
\end{tabular}

Rat bone marrow cells were cultured for forming osteoclast-like MNC as described in Materials and methods. Cultures were performed in the presence or absence of MTX with or without MRS1754. After 4 days of culture, cells were stained for TRAP. ${ }^{* * P}<0.01$ if compared with the control (the first line: without MTX, adenosine, and MRS1754).

Figure 5 Involvement of $A_{2 b} A R$ in the recovery of osteoclastogenesis suppressed by MTX. (a) RT-PCR analysis of the expression of adenosine receptor mRNA. Osteoblasts were isolated from rat calvarias. Total RNA was extracted, and the expression of four different adenosine receptors was examined by RTPCR. The expression of $A_{1}, A_{2 a}$ and $A_{2 b} A R$ was detected in these cells. (b, c) Effect of antagonists. Bone marrow cells were cultured for forming osteoclasts in medium with additions as indicated in the figure. (b) Cells were treated with caffeine, an antagonist for $A_{1} A R, A_{2 a} A R$ and $A_{3} A R$. (c) Cells were treated with MRS1754, a specific antagonist for $A_{2 b} A R$. At 4 days of culture, cells were stained for TRAP and TRAP-positive MNCs were counted. Data show mean values \pm s.e.m. from four cultures. Data were analyzed by Student's $t$-test. ${ }^{* *} P<0.001$ compared with control. ${ }^{\# \# \#} P<0.001$ when compared each pair. N.S.: no significance. Data shown are representative of three independent experiments. (d) Immunohistochemical detection of expression of $A_{2 b} A R$ in distal tibia of AA rats with severe bone destruction. Microscopic observations of distal tibia of AA rats (upper panels) and of control rats (lower panels) 28 days after

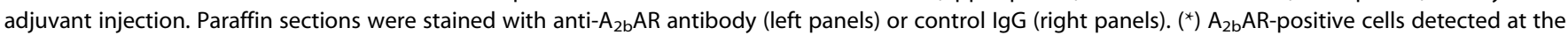
bone-bone marrow interface in the distal tibia of AA rats. (\#) Position of the stromal cells. (control) Normal rabbit lgG. Bars $=100 \mu \mathrm{m}$. 
a

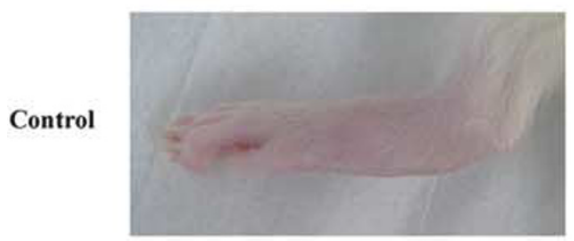

CFA

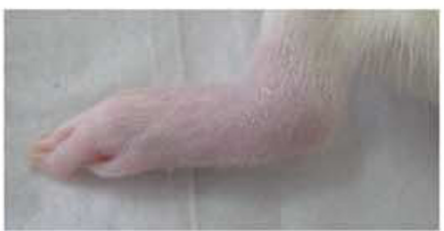

CFA

+ MTX

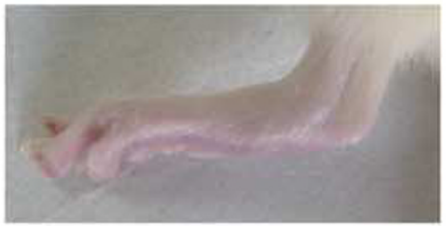

CFA

+ MTX

+ Ad
X-ray
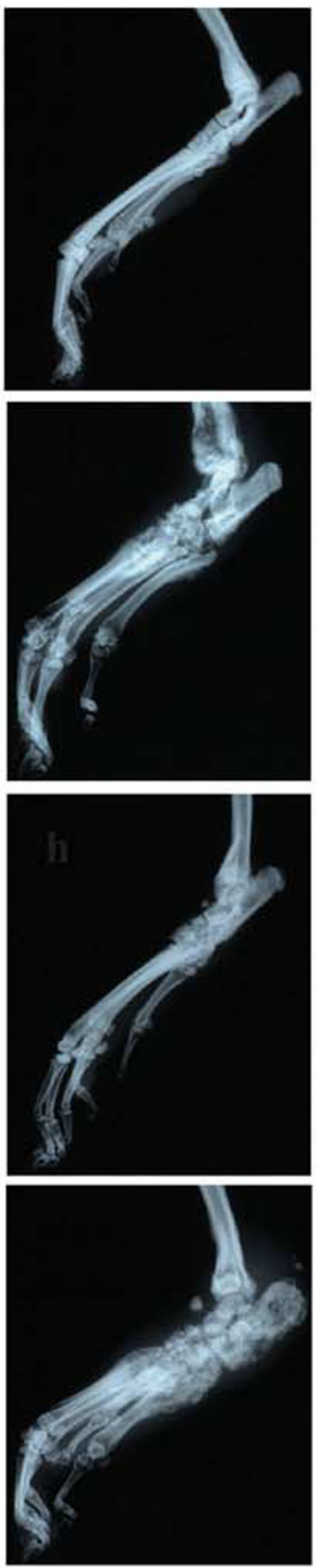

HE
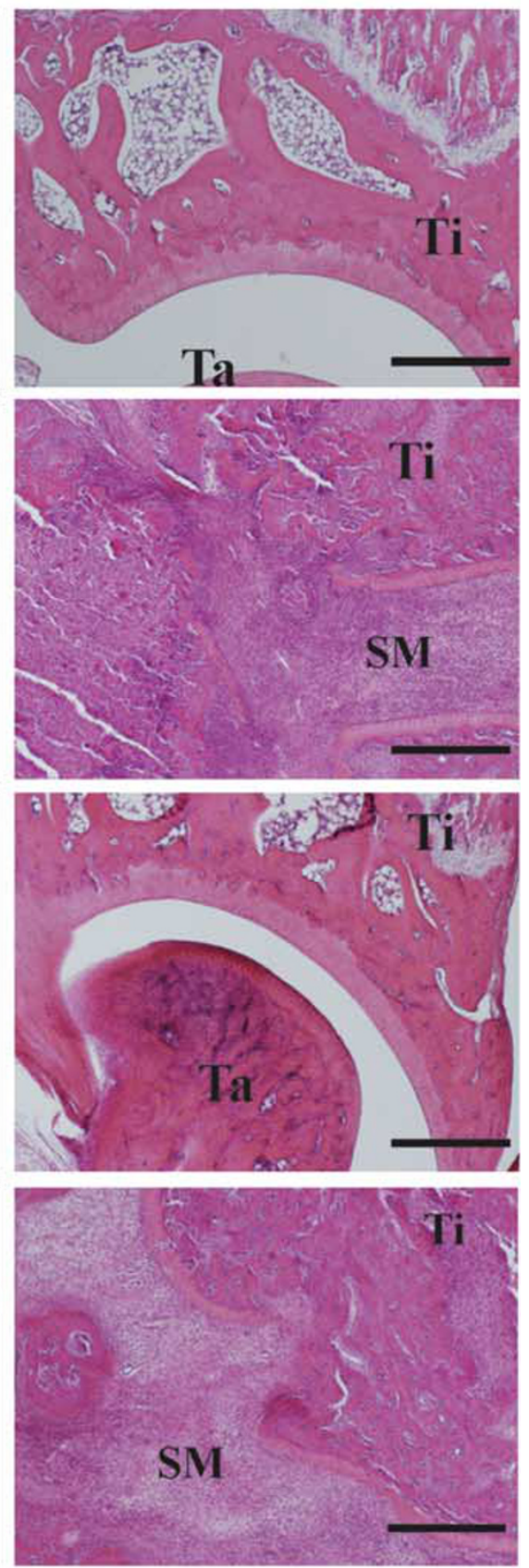

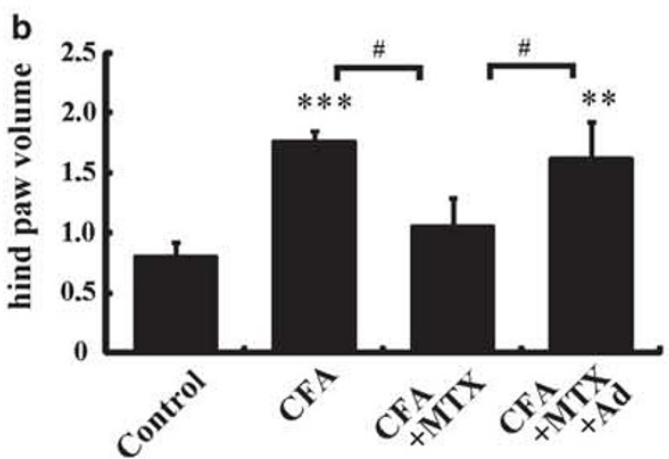


MTX at the late stage of AA did not suppress inflammatory bone destruction. In our experiments of adenosine measurement in the plasma of AA rats, adenosine concentration in plasma was markedly elevated during day 3 to day 10 after adjuvant injection. Although we could not measure the level of adenosine in the synovial fluid because of the technical reasons, the adenosine level is supposed to be very high in the synovial fluid as the plasma level of adenosine increased approximately five-folds from day 3 to day 10 after adjuvant injection. Kara et a ${ }^{26,27}$ reported that $\mathrm{A}_{1} \mathrm{AR}$ has a critical role in osteoclast formation and function. In their study, $A_{1} A R$ knockout mice showed a reduction in osteoclastogenesis as well as suppressing osteoclast function using in vitro bone marrow culture system. Their findings showed a possible involvement of $\mathrm{A}_{1} \mathrm{AR}$ in normal osteoclastogenesis. Montesinos et $a l^{28,29}$ has reported that adenosine acts as the suppressive factor in inflammation. In their model, MTX administration induced a significant level of adenosine; however, its level is $<170 \mathrm{nM}$, which concentration are able to activate only the high-affinity receptors. Several lines of evidence have been reported that $\mathrm{A}_{3}$ receptor have anti-inflammatory and anti-resorptive effects. ${ }^{30}$ In our current study, we have focused on the adenosine-mediated recovery of osteoclastogenesis under the specified condition in which osteoclastogenesis and inflammation are suppressed by MTX. Although more precise quantitative data using micro-CT are required, we could demonstrate that adenosine successfully abolish MTX-induced suppression of bone destruction when it was injected to the joint cavity of AA rats. It would require the high local level of adenosine (of $>40 \mu \mathrm{M}$ ) to express such MTX resistance. We have demonstrated a possible involvement of $A_{2 b} A R$, a low-affinity $A R$, in this process. Adenosine acts osteoblastic stromal cells mainly through $\mathrm{A}_{2 \mathrm{~b}} \mathrm{AR}$ to inhibit OPG production, which allows abundant osteoclastogenesis owing to an increase in the ratio of RANKL/OPG. This mechanism could partly explain the incidence of MTX resistance in some patients with RA. Therefore, simultaneous administration of $\mathrm{A}_{2 \mathrm{~b}} \mathrm{AR}$ antagonist and MTX may improve MTX-based therapy for RA patients. Estimation of adenosine in MTX-resistant patients could open a new diagnostic approach to monitor the progress of RA. Further clinical studies are required to establish a correlation of MTX resistance and the level of adenosine in patience with RA.
Genetic studies of tumors have demonstrated amplification of the dihydrofolate reductase (DHFR) gene in MTXresistant patients. Recently, profound progress at the molecular level has been made in research on MTX-resistant tumors. Mechanisms for explaining resistance to MTX treatment include an increase in dihydrofolate reductase (DHFR) enzyme levels, resulting from DHFR gene overexpression, coupled with impaired intracellular transport of MTX as a consequence of decreased levels of reduced folate carrier (RFC) at the cell membrane. ${ }^{31}$ Serra et $a l^{32}$ demonstrated amplification of the DHFR gene in MTX-resistant osteosarcoma patients. MTX uptake is dependent on RFC. Defective drug uptake in association with decreased RFC is a common mechanism of MTX resistance in many tumor types. Yang et $a l^{33}$ demonstrated that partial promoter methylation and RFC mRNA downregulation were observed in a cell line established from a patient with malignant fibrohistiocytoma. Even if MTX is taken up by target cells, MTX can be polyglutamylated by folylpolyglutamate synthetase (FPGS); polyglutamylated MTX is subjected to extensive cellular retention, which results in maximal cytotoxic activity. Recently, Stark et al ${ }^{34}$ reported aberrant splicing of FPGS in anti-folate resistance in leukemia. Such genetic mutations observed in tumor cells are usually irreversible. In contrast, in our current experiments, adenosine treatment completely abolished MTX-induced suppression of bone destruction in AA rats. This may suggest that MTX resistance in RA is reversible. As RA is an autoimmune disease, it is unlikely that MTX resistance occurs by mutation as observed in MTX-resistant tumors. However, as rheumatoid pannus is considered as a pseudo-tumor, similar molecular mechanisms as tumors may exist in MTX observed in RA. Further studies should be addressed to evaluate a possible involvement of molecular mechanism of MTX resistance similar to tumors.

Several reports have addressed the suppressive effect of MTX on osteoclastogenesis. ${ }^{35,36}$ We also showed, in the current study, that MTX dramatically suppressed RANKL mRNA levels, which is in accord with data of other groups. Adenosine did not affect MTX-induced suppression of RANKL; however, adenosine markedly suppressed expression of OPG mRNA. The decrease in the level of OPG results in an increase in the ratio of RANKL/OPG, causing a recovery of

Figure 6 Adenosine treatment abrogated MTX-induced suppression of bone destruction in AA rats. MTX injections (1 mg/kg/week) were given intraperitoneally beginning on day 3 after the adjuvant injection. Adenosine was injected into the cavity of the left ankle joints as described in Materials and methods. The injections were performed every 3 days beginning on day 3 after the adjuvant injection. PBS was injected into the right ankle joint as an internal vehicle control. (a) Representative photographs (left panels), X-ray images (middle panels), and histologic staining (right panels) of the hind paws. Adjuvant injection induced inflammation and bone destruction. In MTX-treated rats (from day 3 after adjuvant injection), the swelling of ankle joint and bone destruction was dramatically decreased. In contrast, when adenosine was injected into the left ankle joints of AA rats treated with MTX, hind paw swelling and bone destruction at the injected sides was increased (CFA + MTX $+A d)$ to the level seen in adjuvant-treated $A A$ rats (CFA). Ti, tibia; Ta, tarsus; $\mathrm{SM}$, synovial membrane. Bars $=200 \mu \mathrm{m}$. (b) Quantitative demonstration of ankle-joint swelling in AA rats. In AA rats with MTX treatment from day 3 after adjuvant injection, the volume was reduced to the level of controls. In contrast, MTX-induced suppression of joint swelling was canceled by injection of adenosine. Each column and bar represents the mean \pm s.e.m. of four rats. Data were analyzed by Student's $t$-test. ${ }^{\star \star} P<0.01$ and ${ }^{\star \star \star} P<0.001$ when compared with control rats. ${ }^{\#} P<0.05$ when compared each pair. 
osteoclastogenesis. Evans et $a l^{37}$ reported that human osteoblasts produce adenosine and that adenosine has an autocrine or paracrine role in the inhibition of production of OPG protein. We demonstrated that adenosine inhibits gene expression of OPG, possibly through $\mathrm{A}_{2 \mathrm{~b}} \mathrm{AR}$. Evans et al (2006) demonstrated that endogenous adenosine acts on osteoblastic cells to increase IL6 release. It is also possible to consider that adenosine acts through upregulation of IL6 in our experimental systems to augment osteoclastogenesis.

MRS1754, a highly selective antagonist for $A_{2 b} A R$, might be useful in overcoming MTX resistance in RA patients. Extracellular ATP is known to support osteoclast function through formation of the sealing zone and the secretion of lytic granules required for osteoclast function via P2X7 nucleotide receptors. ${ }^{38,39}$ Extracellular ATP is promptly converted into AMP and adenosine by the sequential action of CD39 (NTPDase) and CD73 (Ecto5 ${ }^{\prime}$ NTase), which results in an increase in the local level of adenosine to the $10-100 \mu \mathrm{M}$ level. In the MTX-resistant conditions in inflammatory bone destruction, a high level of extracellular adenosine produced in the inflammatory sites could inhibit production of OPG, and allows formation of abundant osteoclasts and consequent severe bone destruction.

Recently, anti-TNF $\alpha$ therapy has been shown to be successful in MTX-resistant RA patients; however, severe side effects of this treatment were also reported. ${ }^{10}$ Blockage of adenosine- $\mathrm{A}_{2 \mathrm{~b}} \mathrm{AR}$ could provide a milder, but effective, therapy with minimal side effects in comparison to an intensive therapy such as anti-TNF $\alpha$.

We found that MTX-induced suppression of bone destruction was abolished by extracellular adenosine through suppression of OPG expression. The uptake of extracellular adenosine via adenosine- $\mathrm{A}_{2 \mathrm{~b}} \mathrm{AR}$ receptor system appears to explain MTX resistance in RA. Careful utilization of MTX considering the dose is quite important ${ }^{40}$ and parallel utility with other safer medicine is considered to be more effective. Our study provides basic information concerning the development of a novel therapy to overcome MTX resistance in inflammatory bone destruction by use of specific antagonists for ARs.

\section{ACKNOWLEDGEMENTS}

This research was supported in part by the Grant-in-Aid for Scientific Research (C) and (B) of Japan Society for the Promotion of Science (JSPS) (project no. 19592116 and no. 21390492). We acknowledge emeritus Professor T lijima for encouragements. We thank Dr Eric Milner for proofreading this manuscript.

\section{DISCLOSURE/CONFLICT OF INTEREST}

The authors declare no conflict of interest.

1. Shimizu S, Shiozawa S, Shiozawa K, et al. Quantitative histologic studies on the pathogenesis of periarticular osteoporosis in rheumatoid arthritis. Arthritis Rheum 1985;28:25-31.

2. Takayanagi H. Osteoimmunology: shared mechanisms and crosstalk between the immune and bone systems. Nat Rev Immunol 2007;7:292-304.
3. Singer FR. Paget disease: when to treat and when not to treat. Nat Rev Rheumatol 2009;5:483-489.

4. Farber S, Diamond LK. Temporary remissions in acute leukemia in children produced by folic acid antagonist, 4-aminopteroyl-glutamic acid. N Engl J Med 1948;238:787-793.

5. Geiser CF, Bishop Y, Jaffe N III, et al. Adverse effects of intrathecal methotrexate in children with acute leukemia in remission. Blood 1975;45:189-195.

6. Thompson PA, Murry DJ, Rosner GL, et al. Methotrexate pharmacokinetics in infants with acute lymphoblastic leukemia. Cancer Chemother Pharmacol 2007;59:847-853.

7. Weinblatt ME, Coblyn JS, Fox DA, et al. Efficacy of low-dose methotrexate in rheumatoid arthritis. N Engl J Med 1985;312:818-822.

8. Buckley LM, Leib ES, Cartularo KS, et al. Effects of low dose methotrexate on the bone mineral density of patients with rheumatoid arthritis. J Rheumatol 1997;24:1489-1494.

9. Choi HK, Seeger JD, Kuntz KM. A cost-effectiveness analysis of treatment options for patients with methotrexate-resistant rheumatoid arthritis. Arth Rheumat 2000;43:2316-2327.

10. Barbieri $M$, Wong JB, Drummond $M$. The cost effectiveness of infliximab for severe treatment-resistant rheumatoid arthritis in the UK. Pharmacoeconomics 2005;23:607-618.

11. Romas E, Gillespie MT. Inflammation-induced bone loss: can it be prevented? Rheum Dis Clin North Am 2006;32:759-773.

12. O'Brien CA. Control of RANKL gene expression. Bone 2010;46:911-919.

13. Boyle WJ, Simonet WS, Lacey DL. Osteoclast differentiation and activation. Nature 2003;423:337-342.

14. Suda T, Takahashi N, Udagawa N, et al. Modulation of osteoclast differentiation and function by the new members of the tumor necrosis factor receptor and ligand families. Endocr Rev 1999;20: 345-357.

15. Leibbrandt A, Penninger JM. RANK/RANKL: regulators of immune responses and bone physiology. Ann N Y Acad Sci 2008;1143:123-150.

16. Jacobson KA, Gao ZG. Adenosine receptors as therapeutic targets. Nat Rev Drug Discov 2006;5:247-264.

17. Kukita A, Kukita T, Hata K, et al. Heat-treated osteoblastic cell (ROS17/ 2.8)-conditioned medium induces the formation of osteoclast-like cells. Bone Miner 1993;23:113-127.

18. Kukita A, Kukita $\mathrm{T}$, Shin $\mathrm{JH}$, et al. Induction of mononuclear precursor cells with osteoclastic phenotypes in a rat bone marrow culture system depleted of stromal cells. Biochem Biophys Res Commun 1993;196:1383-1389.

19. Takahashi N, Akatsu T, Udagawa N, et al. Osteoblastic cells are involved in osteoclast formation. Endocrinology 1988;123:2600-2602.

20. Kuratani T, Nagata K, Kukita T, et al. Induction of abundant osteoclastlike multinucleated giant cells in adjuvant arthritic rats with accompanying disordered high bone turnover. Histol Histopathol 1998;13:751-759.

21. Nakamura $T$, Kukita $T$, Shobuike $T$, et al. Inhibition of histone deacetylase suppresses osteoclastogenesis and bone destruction by inducing IFN-beta production. J Immunol 2005;175:5809-5816.

22. Sullivan GW, Rieger JM, Scheld WM, et al. Cyclic AMP-dependent inhibition of human neutrophil oxidative activity by substituted 2propynylcyclohexyl adenosine $A(2 A)$ receptor agonists. Br J Pharmacol 2001;132:1017-1026.

23. Hasko G, Cronstein BN. Adenosine: an endogenous regulator of innate immunity. Trends Immunol 2004;25:33-39.

24. Martin C, Leone $M$, Viviand $X$, et al. High adenosine plasma concentration as a prognostic index for outcome in patients with septic shock. Crit Care Med 2000;28:3198-3202.

25. Cronstein BN. Adenosine, an endogenous anti-inflammatory agent. J Appl Physiol 1994;76:5-13.

26. Kara FM, Chitu V, Sloane J, et al. Adenosine A1 receptors (A1Rs) play a critical role in osteoclast formation and function. FASEB J 2010;24:2325-2333.

27. Kara FM, Doty SB, Boskey $A$, et al. Adenosine $A(1)$ receptors regulate bone resorption in mice: adenosine $A(1)$ receptor blockade or deletion increases bone density and prevents ovariectomy-induced bone loss in adenosine $\mathrm{A}(1)$ receptor-knockout mice. Arthritis Rheum 2010;62:534-541.

28. Montesinos $M$, Desai $A$, Delano $D$, et al. Adenosine $A_{2 A}$ or $A_{3}$ receptors are required for inhibition of inflammation by metotrexate and its analogue MX-68. Arthritis Rheum 2003;48:240-247. 
29. Montesinos M, Yap J, Desai A, et al. Reversal of the antiinflammatory effects of methotrexate by the nonselective adenosine receptor antagonists teophillin and caffein: evidence that the antiinflammatory effects of methotrexate are mediated via multiple adenosine receptors in rat adjuvant arthritis. Arthritis Rheum 2000;43:659-663.

30. Baharav E, Bar-Tehuda S, Madi L, et al. Antiinflammatory effect of $A_{3}$ adenosine receptor agonists in murine autoimmune arthritis model. J Rheumatol 2005;32:469-476.

31. Guo W, Healey JH, Meyers PA, et al. Mechanisms of methotrexate resistance in osteosarcoma. Clin Cancer Res 1999;5:621-627.

32. Serra M, Reverter-Branchat G, Maurici D, et al. Analysis of dihydrofolatereductase and reduced folate carrier gene status in relation to methotrexate resistance in osteosarcoma cells. Ann Onco 2004;15:151-160.

33. Yang $\mathrm{R}$, Li WW, Hoang $\mathrm{BH}$, et al. Quantitative correlation between promoter methylation and messenger RNA levels of the reduced folate carrier. BMC Cancer 2008;8:124.

34. Stark M, Wichman C, Avivi I, et al. Aberrant splicing of folylpolyglutamate synthetase as a novel mechanism of antifolate resistance in leukemia. Blood 2009;113:4362-4369.
35. Lee CK, Lee EY, Chung SM, et al. Effects of disease-modifying antirheumatic drugs and antiinflammatory cytokines on human osteoclastogenesis through interaction with receptor activator of nuclear factor kappaB, osteoprotegerin, and receptor activator of nuclear factor kappaB ligand. Arthritis Rheum 2004;50:3831-3843.

36. Suematsu A, Tajiri Y, Nakashima T, et al. Scientific basis for the efficacy of combined use of antirheumatic drugs against bone destruction in rheumatoid arthritis. Mod Rheumatol 2007;17:17-23.

37. Evans BA, Elford C, Pexa A, et al. Human osteoblast precursors produce extracellular adenosine, which modulates their secretion of IL- 6 and osteoprotegerin. J Bone Miner Res 2006;21:228-236.

38. Hazama R, Qu X, Yokoyama $\mathrm{K}$, et al. ATP-induced osteoclast function the formation of sealing-zone like structure and the secretion of lytic granules via microtubule-deacetylation under the control of Syk. Genes Cells 2009;14:871-884.

39. Armstrong S, Pereverzev A, Dixon SJ, et al. Activation of P2X7 receptors causes isoform-specific translocation of protein kinase $C$ in osteoclasts. J Cell Sci 2009;122(Part 1):136-144.

40. Cronstein BN. Low-dose methotrexate: a mainstay in the treatment of rheumatoid arthritis. Pharmacol Rev 2005;57:163-172. 